\title{
A Regional Metabolic Contrast Method for the Study of Brain Pathology
}

\author{
Bernard W. Agranoff, MD, and Kirk A. Frey, SB
}

The use of 2-deoxyglucose for the measurement of regional cerebral glucose utilization in both animals and humans has gained widespread application. An attractive aspect of regional brain glucose metabolism is its apparent correlation with functional activity in neurons. Animal experiments suggest that this relationship is altered in brain lesions and that barbiturate anesthesia may be employed in the detection of brain regions where uncoupling of metabolism and neuronal function has occurred. A functional metabolic contrast technique is described that will permit determination of regional metabolism that is specifically attributable to neuronal activity. Areas of potential clinical application are discussed.

Agranoff BW, Frey KA: A regional metabolic contrast method for the study of brain pathology. Ann Neurol 15(suppl):S93-S97, 1984

The carbon-14-labeled 2-deoxy-D-glucose $\left(\left[{ }^{14} \mathrm{C}\right] 2 \mathrm{DG}\right)$ technique of Sokoloff and co-workers [28] has led to the characterization of regional cerebral glucose metabolism in animals under a variety of experimental conditions. Imposed experimental variables as diverse as sensory stimulation, systemic drug administration, and cortical penicillin application have each revealed alterations in glucose metabolic utilization patterns that reflect characteristic changes in neuronal activity [27]. Thus, the $\left[{ }^{14} \mathrm{C}\right] 2 \mathrm{DG}$ method has enjoyed widespread application as a technique for determining neuronal responses to physiological and pathophysiological actions. In comparison with previous techniques, the method has great advantage in that the entire brain can be examined simultaneously in autoradiographic sections with considerable histological detail.

\section{Relation of 2DG Uptake to Synaptic \\ Transmission and Its Modulation}

The inferred correlation between neuronal activity and regional glucose metabolism is further strengthened when one considers the detailed anatomical distribution of carbon-14-labeled 2-deoxyglucose 6-phosphate $\left(\left[{ }^{14} \mathrm{C}\right] 2 \mathrm{DG}-6-\mathrm{P}\right)$ in autoradiographic experiments. Gray matter regions with the greatest density of synaptic connections have relatively higher rates of glucose utilization than those that are predominantly cellular, and both have metabolic rates much greater than white matter in awake animals $[25,28]$. This hierarchy of glucose metabolism is best demonstrated in brain regions where the various neuronal elements are anatomically stratified. For example, in neocortex the density of labeling following $\left[{ }^{14} \mathrm{C}\right] 2 \mathrm{DG}$ utilization is greatest over layer IV, a region in which afferent thalamic fibers synapse on cortical neurons [3, 10]. Labeling in the hippocampus is heaviest in the synaptic molecular layer and progressively less intense over the pyramidal cell layer, the stratum oriens, and the fornix.

The correlation of regional $\left[{ }^{14} \mathrm{C}\right] 2 \mathrm{DG}$ utilization with neuronal activity and the local distributions observed over dense synaptic areas suggest that the metabolism underlying synaptic transmission plays a major role in the coupling of glucose utilization and neuronal activity. In vitro experiments using the posterior pituitary have verified the sensitivity of presynaptic glucose utilization to depolarization and have further demonstrated the critical role of sodium-potassium adenosine triphosphatase in this coupling [17]. At the neural synapse, however, this metabolic response to presynaptic activation must be considered within the context of the polarity and magnitude of the postsynapric response as well. Presynaptic activity can be assumed to be metabolically costly regardless of the type of synapse involved. Postsynaptic responses, on the other hand, should result in additional energy utilization at excitatory synapses, while at inhibitory synapses there may be no change in energy consumption or even a decrease in metabolic demand with presynaptic stimulation. Thus, changes in overall synaptic metabolism should correlate positively with neuronal activity at excitatory synapses, while the metabolism of inhibitory synapses could increase or decrease with activation.

Experiments in anesthetized animals have demon-
From the Neuroscience Laboratory, Mental Health Research Institute, University of Michigan, Ann Arbor, MI 48109.
Address reprint requests to Dr Agranoff, Neuroscience Laboratory Building, University of Michigan, 1103 East Huron Ave, Ann Arbor, MI 48109. 
strated that electrical stimulation of known inhibitory pathways can result in an increase in synaptic 2DG accumulation [1], while other studies have demonstrated profound attenuation of glucose metabolism when postsynaptic inhibitory receptors are activated by systemic or local administration of gammaaminobutyric acid agonists $[3,9,20]$. The net metabolic response at inhibitory synapses may depend on a number of factors, including the relative contributions of presynaptic and postsynaptic elements based on their surface areas and cytoplasmic volumes [26], the level of postsynaptic excitation from other sources, and still other intervening or modulating mechanisms. Thus, it may be speculated that 2DG metabolic studies should correlate well with direct excitatory events in the brain, while caution should be exercised in the functional interpretation of results in cases in which a major contribution from synaptic inhibition is expected.

\section{Effects of Barbiturates in Brain Lesions}

Results obtained in our laboratory [6] suggest further caveats in the use of $2 D G$ for the estimation of neuronal function following brain lesions. When rats are studied one week following striatal ibotenic acid lesions using the $\left[{ }^{14} \mathrm{C}\right] 2 \mathrm{DG}$ autoradiographic technique, a zone of decreased labeling is observed in the striatum corresponding to the lesion site (Figure, part A). Brain regions immediately postsynaptic to the lesion demonstrate increased isotope uptake, as seen in the pars reticulata of the substantia nigra (Figure, part B). Our original interpretation of these findings was that the 20\% decrease in 2DG uptake within the lesion was attributable to the loss of the intrinsic neurons, and that the residual labeling represented metabolism in glia and presynaptic terminals of extrinsic neurons known to be spared in ibotenic or kainic acid lesions [24]. The increased labeling of postsynaptic regions could be attributable to disinhibition following the removal of the predominantly gamma-aminobutyric acid-mediated striatal innervation. In order to test the latter hypothesis, we studied lesioned rats that had been pretreated with the gamma-aminobutyric acid agonist muscimol. Contrary to our expectations, the denervated substantia nigra was even less sensitive to metabolic suppression by muscimol than the contralateral, intact nigra. Additionally, the lesioned striatum was less affected by the muscimol treatment than the control side. Rats pretreated with anesthetic doses of pentobarbital demonstrated the same resistance to metabolic suppression observed with muscimol, a result that indicated that the residual asymmetrical 2DG labeling (Figure, parts $C$ and $D$ ) was unrelated to synaptic neuronal activity.

Additional autoradiographic studies were performed with $\left[{ }^{14} \mathrm{C}\right]$ iodoantipyrine to measure regional blood flow. The results of these studies confirm that perfusion of the lesioned striatum is relatively preserved in excitatory neurotoxin lesions and suggest that the refractory response of the damaged areas to barbiturates is not a consequence of decreased drug delivery.

Another feature that is common to both the striatal lesion and its projection sites is the presence of degenerating neuronal structures associated with an infiltrate of phagocytic cells. We therefore interpret the results to indicate that the appearance of barbiturateinsensitive 2DG labeling is a reflection of the cellular inflammatory process and may well be characteristic of the subacute cerebral response to a variety of insults, including ischemic infarction and encephalitis. When cerebral metabolism was studied with a 10 minute $\left[{ }^{14} \mathrm{C}\right]$ glucose pulse, a much greater impairment of isotope accumulation was observed in the lesion (a $50 \%$ decrease) (Figure, part E), in comparison to the 2DG data. An attractive explanation for both the barbiturate resistance and the difference observed between labeled glucose and 2DG is the presence of anaerobic glycolysis within the inflammatory infiltrate. Studies in both neutrophils and monocytes have demonstrated dramatic increases in metabolism during phagocytosis $[22,23]$. Basal adenosine triphosphate generation in these cells is largely via anaerobic glycolysis, even in the presence of high oxygen concentrations. Phagocytosis and lysosomal activation are associated with both increased oxygen consumption and increased glucose utilization via the hexose monophosphate shunt pathway to produce the reduced nicotinamide-adenine dinucleotide phosphate that is presumed necessary for peroxide synthesis. Thus, we would anticipate both increased glycolytic activity and increased oxygen utilization in cerebral phagocytes following injury.

\section{Potential Application in Studies of Cerebral Infarction and Other Disorders}

With the recent advent of positron emission tomographic (PET) methods capable of imaging the human brain and the development of rapid radiochemical syntheses of positron-emitting glucose analogs, the 2DG technique has been applied to a variety of clinical research problems. The extension of the beta emitterbased autoradiographic method to emission tomography presents advantages beyond the obvious fact that it permits us to study regional metabolism of the human brain. PET technology also permits us to perform repetitive studies in the same subject. The problem of inherent variability among subjects is thereby eliminated. The design of PET protocols that compare results obtained in the same subject under varying conditions is particularly attractive. The principle of using a 


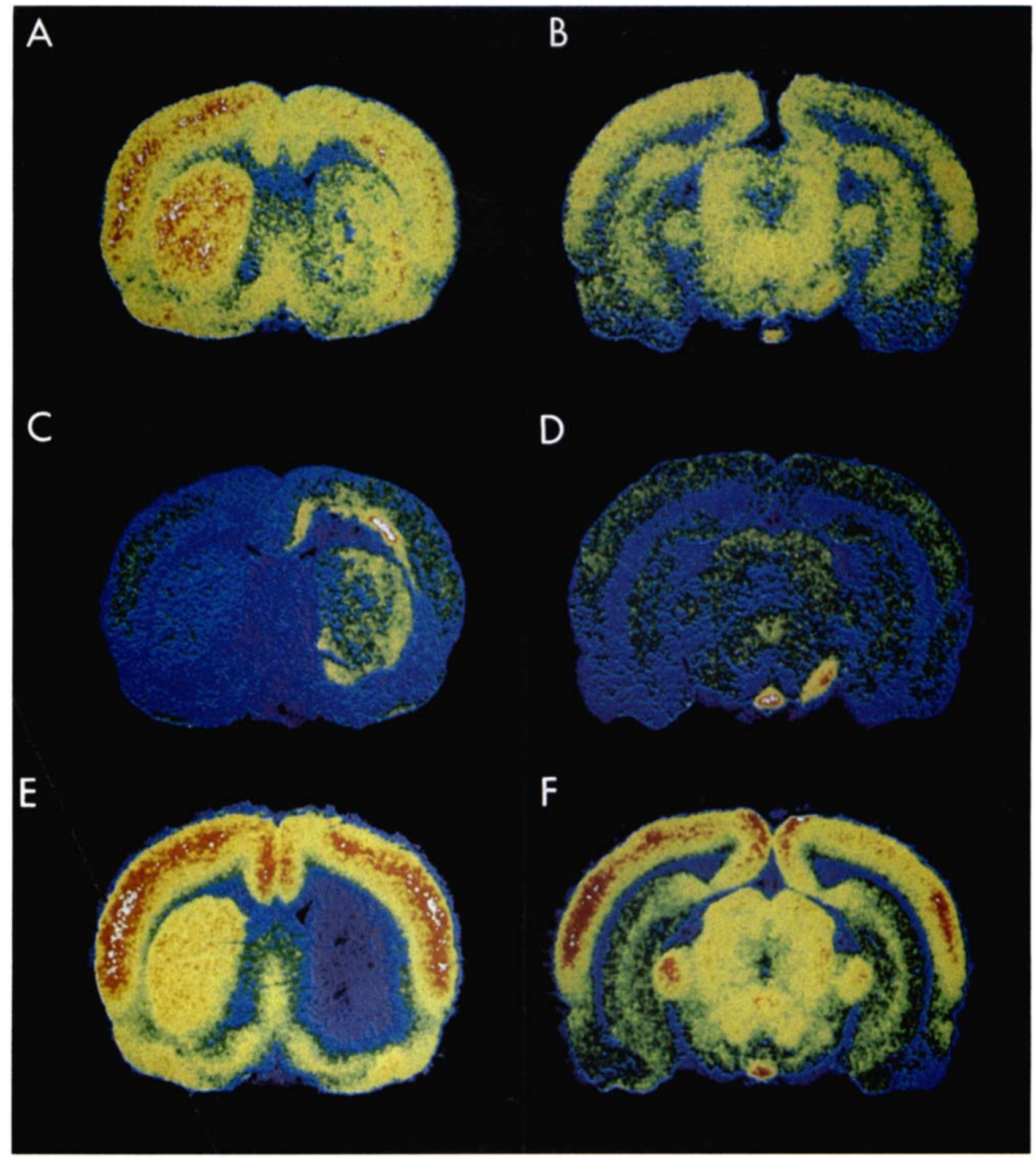

Color-coded autoradiographs of coronal sections of rat brain seven days following ibotenic acid lesion of the right striatum. $(A, B)$ Uptake of carbon-14-labeled 2-deoxyglucose $\left(\left(^{14} \mathrm{C} D \mathrm{D} G\right)\right.$ in awake animal. Tracer accumulation is decreased in the lesion $(A)$ and increased in the denervated substantia nigra (B). (C, D) Uptake of $\left({ }^{14} C\right) 2 D G$ in animal anesthetized with barbiturate. Botb the lesion $(C)$ and the ipsilateral substantia nigra $(D)$ appear resistant to the metabolic suppression observed following barbiturate administration. $(E, F)\left({ }^{14} \mathrm{C}\right)$ Glucose labeling. Note the greater

decrease in labeling in the lesion (E) than was observed with $2 D G(A)$. No asymmetry is observed in the substantia nigra $(F)$. Film densities were converted to pseudocolor images using a computer-assisted scanning densitometry facility at the neuroscience laboratories of the University of Michigan and the method described by Goochee and co-workers $\{7\}$. Areas of bighest isotope uptake are white and red. Yellow, green, blue, and violet correspond to progressively decreasing concentrations of isotope in the tissue underlying the autoradiographic film. 
subject as his or her own control has in fact been demonstrated previously in animal studies with hydrogen3-labeled and carbon-14-labeled deoxyglucose, using a sequential double-label method [2]. In addition, the ability to follow regional time-activity curves using sensitive tomographs with good temporal resolution makes PET 2DG studies much less dependent upon assumed kinetic constants derived from control populations [8]. Thus, while the spatial resolution of PET 2DG scans is well below the microscopic level achieved in autoradiographic studies in animals, it possesses distinct advantages. For example, the comparison of sequential PET studies with and without barbiturate anesthesia (a barbiturate contrast method) will allow the determination of regional metabolism directly coupled with neuronal function. This procedure should provide greater specificity in the interpretation of results than that obtained from a single scan. Additionally, the regional distribution of barbiturateinsensitive metabolism may itself be of interest, as suggested by the previously described animal study.

The results in ibotenate-treated animals appear to be directly applicable to a number of clinical problems using the new noninvasive techniques. In particular, questions regarding the pathophysiology of brain ischemia and infarction can be addressed. On the basis of information obtained thus far, it is now clear that energy metabolism of the brain and cerebral blood flow can be dissociated in pathological states $[13,14,16,19$, $29,30]$. Conditions under which there is regional ischemia in the presence of relatively normal rates of brain metabolism (poverty perfusion) as well as those in which there is increased cerebral blood flow relative to energy metabolism (luxury perfusion) have been documented. Increased oxygen utilization following stroke has been interpreted to indicate peroxidative and otherwise energy-consuming steps associated with the destruction and removal of damaged tissue by reactive cells [29]. An increase in regional glucose utilization [13] has been interpreted to indicate increased glycolytic activity, which is thought to be relatively minor in the energy economy of normal brain tissue. As discussed previously, both of these metabolic alterations have been observed in the inflammatory cells that are expected in response to cerebral infarction. A sequential contrast study in a patient with a recent stroke using positron-labeled 2DG or molecular oxygen in the presence and in the absence of barbiturate should clearly distinguish normally functioning neuronal tissue from other metabolic activity, which should be insensitive to the effects of barbiturate anesthesia.

The 2DG-barbiturate animal experiments reviewed here indicate not only that the primary site of injury is refractory to the suppressive effect that barbiturates have on normal neuropil but also that changes occur in afferent projections from the damaged tissue. Present evidence suggests that these latter changes may, like the primary site, reflect reactive and resorptive processes. We can thus expect that anesthetized subjects with an infarcted cortical zone will also manifest increased 2DG utilization in the thalamus and other subcortical regions.

The contrast principle espoused here may prove of interest in a number of other disorders. For example, the nature of the hypometabolic region surrounding an epileptic focus $[5,11]$ is presently unexplained. If it represents, in part, replacement of normal functioning neural tissue with reactive cells or gliotic scar, this change should be demonstrable by a metabolic contrast study.

It will also be of great interest to learn whether the comparison of regional brain glucose utilization in the awake and in the sedated state varies as a function of age. While it is known that the brain decreases in size with age [4] and that cerebral blood flow decreases as well $[15,18]$, there is no unanimity regarding the question of whether or not systemic changes in energy metabolism also occur. While evidence has been reported for a decrease in glucose metabolism in cortical tissues [12], there does not appear to be agreement regarding a commensurate decrease in oxygen utilization. This possible discrepancy could be explained in a number of ways, for example, by a switch to an alternative fuel, such as to ketone body metabolism. On the other hand, the discrepancy could reflect increased microsomal or lysosomal oxygen metabolism unrelated to adenosine triphosphate generation.

The use of anesthesia carries with it possible risk to the subject, so that it will be important to learn whether deep sedation is required to produce a substantial metabolic decrement. The recent introduction of improved synthesis of $\left[{ }^{11} \mathrm{C}\right] 2 \mathrm{DG}$ makes it feasible to perform sequential studies in a single day [21], whereas with fluorine-18-labeled fluorodeoxyglucose ( ${ }^{18} \mathrm{FDG}$ ) at least 9 to 10 hours are required between studies to allow the first dose of ${ }^{18}$ FDG to decay sufficiently before the second study can be performed. For data analysis of contrast studies, it is important that the same tomographic slice levels be compared and that comparable brain regions be accurately identified in scan regions subjected to quantitative analysis. Ideally, the head would be held immobile or would be reinserted into a head-holder with great precision, so that accurate subtraction images could be made. Recent progress in the subtraction of PET images in oxygen-15 studies gives encouragement that this goal may eventually be achieved. The use of $\left[{ }^{11} \mathrm{C}\right] 2 \mathrm{DG}$, together with advances in PET image registration, will unquestionably make the contrast method even more powerful in identifying pathological brain tissue. 
Supported by Grant NS 15655 from the National Institutes of Health. K. A. F. is a trainee of NIH Grant 1 T32 GM07863.

The authors acknowledge with thanks the work of Dr George Dauth in implementing the scarining densitometry system.

\section{References}

1. Ackerman RF, Finch DM, Babb TL, Engel J Jr: Hippocampal recurrent inhibition: decreased pyramidal cell firing with increased metabolism in the pyramidal cell layer demonstrated by the 2-deoxyglucose autoradiographic technique. Soc Neurosci Abstr 7:457, 1981

2. Altenau LL, Agranoff BW: A sequential double-label 2deoxyglucose method for measuring regional cerebral metabolism. Brain Res 153:375-381, 1978

3. Collins RC: Intracortical localization of 2-deoxyglucose metabolism: on-off metabolic columns. In Passonneau JV, Hawkins RA, Lust WD, Welsh FA (eds): Cerebral Metabolism and Neural Function. Baltimore, Williams \& Wilkins Company, 1980, pp 338-351

4. Davis PJM, Wright EA: A new method for measuring cranial cavity volume and its application to the assessment of cerebral atrophy at autopsy. Neuropathol Appl Neurobiol 3:341, 1977

5. Engel J Jr, Kuhl DE, Phelps ME, Crandall PH: Comparative localization of epileptic foci in partial epilepsy by PCT and EEG. Ann Neurol 12:529-537, 1982

6. Frey KA, Agranoff BW: Barbiturate-enhanced detection of brain lesions by carbon-14-labeled 2-deoxyglucose autoradiography. Science 219:879-881, 1983

7. Goochee C, Rasband W, Sokoloff L: Computerized densitometry and color coding of $\left[{ }^{14} \mathrm{C}\right]$ deoxyglucose autoradiographs Ann Neurol 7:359-370, 1980

8. Huang S-C, Phelps ME, Hoffman EJ, et al: Noninvasive determination of local cerebral metabolic rate of glucose in man. Am J Physiol 238:E69-E82, 1980

9. Keily PAT, McCulloch J: Effects of the putative GABAergic agonists, muscimol and THIP, upon local cerebral glucose utilisation. J Neurochem 39:613-624, 1982

10. Kennedy C, Des Rosiers MH, Sakurada O, et al: Metabolic mapping of the primary visual system of the monkey by means of the autoradiograph $\left[{ }^{14} \mathrm{C}\right]$ deoxyglucose technique. Proc Nat Acad Sci USA 73:4230-4234, 1976

11. Kuhl DE, Engel J Jr, Phelps ME, Selin C: Epileptic patterns of ocal cerebral metabolism and perfusion in humans determined sy emission computed tomography of ${ }^{18} \mathrm{FDG}$ and ${ }^{13} \mathrm{NH}_{3}$. Ann Neurol 8:348-360, 1980

12. Kuhl DE, Mecter EJ, Riege WH, Phelps ME: Effects of human aging on patterns of local cerebral glucose utilization determined sy the $\left[{ }^{18} \mathrm{~F}\right]$ fluorodeoxyglucose method. J Cerebral Blood Flow Metab 2:163-171, 1982

13. Kuhl DE, Phelps ME, Kowell AP, et al: Effects of stroke on local zerebral metabolism and perfusion: mapping by emission computed tomography of ${ }^{18} \mathrm{FDG}$ and ${ }^{13} \mathrm{NH}_{3}$. Ann Neurol 8:47-60, 1980
14. Lassen NA: The luxury-perfusion syndrome and its possible relation to acute metabolic acidosis localised within the brain. Lancet 2:1113-1115, 1966

15. Lebrun-Grandie P, Baron J-C, Soussaline F, et al: Coupling between regional blood flow and oxygen utilization in the normal human brain. Arch Neurol 40:230-236, 1983

16. Lenzi GL, Frackowiak RSJ, Jones T: Cerebral oxygen metabolism and blood flow in human cerebral ischemic infarction. $J$ Cerebral Blood Flow Metab 2:321-335, 1982

17. Mata M, Fink DJ, Gainer H, et al: Activity-dependent energy metabolism in rat posterior pituitary primarily reflects sodium pump activity. J Neurochem 34:213-215, 1980

18. Melamed E, Lavy S, Bentin S, et al: Reduction in regional cerebral blood flow during normal aging in man. Stroke 11:31-35, 1980

19. Olsen TS, Larsen B, Skriver EB, et al: Focal cerebral hyperemia in acute stroke: incidence, pathophysiology and clinical significance. Stroke 12:598-606, 1981

20. Palacios JM, Kuhar MJ, Rapoport SI, London ED: Effects of $\gamma$ aminobutyric acid agonist and antagonist drugs on local cerebral glucose utilization. J. Neurosci 2:853-860, 1982

21. Reivich $M$, Alavi $A$, Wolf $A$, et al: Use of 2-deoxy-D[1${ }^{11} \mathrm{C}$ gglucose for the determination of local cerebral glucose metabolism in humans: variation within and between subjects. J Cerebral Blood Flow Metab 2:307-319, 1982

22. Sbarra AJ, Karnovsky ML: The biochemical basis of phagocytosis. I. Metabolic changes during the ingestion of particles by polymorphonuclear leukocytes. J Biol Chem 234:1355-1362, 1959

23. Sbarra AJ, Strauss RR (eds): The Reticuloendothelial System. A Comprehensive Treatise, Vol 2: Biochemistry and Metabolism. New York, Plenum Press, 1980

24. Schwarcz R, Hokfelt $T$, Fuxe $K$, et al: Ibotenic acid-induced neuronal degeneration: a morphological and neurochemical study. Exp Brain Res 37:199-216, 1979

25. Schwartz WJ, Sharp FR: Autoradiographic maps of regional brain glucose consumption in resting, awake rats using $\left[{ }^{14} \mathrm{C}\right] 2-$ deoxyglucose. J Comp Neurol 177:335-360, 1978

26. Schwartz WJ, Smith CB, Davidsen L, et al: Metabolic mapping of functional activity in the hypothalamo-neurohypophysial system of the rat. Science 205:723-725, 1979

27. Sokoloff $\mathbf{L}$ : Localization of functional activity in the central nervous system by measurement of glucose utilization with radioactive deoxyglucose. J Cerebral Blood Flow Metab 1:7-36, 1981

28. Sokoloff L, Reivich $M$, Kennedy $C$, et al: The $\left[{ }^{14} \mathrm{C}\right] \mathrm{de}-$ oxyglucose method for the measurement of local cerebral glucose utilization: theory, procedure, and normal values in the conscious and anesthetized albino rat. $\mathrm{J}$ Neurochem 28: 897-916, 1977

29. Wise RJS: Positron emission tomography in the study of cerebral ischaemia. J Cerebral Blood Flow Metab 2(suppl):S87-S89, 1982

30. Wise RJS, Bernardi S, Frackowiak RSJ, et al: Serial observations on the pathophysiology of acute stroke: the transition from ischaemia to infarction as reflected in regional oxygen extraction. Brain 106:197-222, 1983 